\title{
Frações de fósforo em Latossolos ${ }^{(1)}$
}

Meire Adélia da Silva(2), Júlio César Azevedo Nóbrega ${ }^{(2)}$, Nilton Curi(2), José Oswaldo Siqueira(2), João José Granate de Sá e Melo Marques ${ }^{(2)}$ e Paulo Emilio Ferreira da Motta ${ }^{(3)}$

Resumo - O objetivo deste trabalho foi verificar se o histórico de uso, adubação fosfatada, inoculação micorrízica e cultivo com braquiária e soja, em casa de vegetação, alteram as frações de P em três Latossolos. Foi determinado o P ligado a $\mathrm{Al}$ (P-Al), Fe (P-Fe) e Ca (P-Ca) e as frações de $\mathrm{P}$ separadas com resina, $\mathrm{NaHCO}_{3}, \mathrm{NaOH}, \mathrm{HCl}$ e digestão com $\mathrm{H}_{2} \mathrm{SO}_{4}$ e $\mathrm{H}_{2} \mathrm{O}_{2}$. As frações orgânicas e inorgânicas foram separadas por autoclavagem. No Latossolo Amarelo coeso (LAx) e Latossolo Vermelho ácrico (LVw), o P-Al, P-Fe e P-Ca foram maiores nos solos cultivados, e no Latossolo Vermelho distroférrico (LVdf) o P-Al e P-Fe foram maiores nos solos não cultivados, tendências não alteradas pelo cultivo com braquiária e soja. A adição de Pelevou principalmente as frações inorgânicas lábeis (Pi-resina e Pi-NaHCO ${ }_{3}$ ) e moderadamente lábil (Pi-NaOH). Solos anteriormente cultivados tiveram menos P nas frações orgânicas do que os não cultivados. Nos solos com braquiária, verificou-se menos $\mathrm{P}$ nas frações pouco lábeis e mais nas frações mais lábeis. O efeito da micorriza foi menor e menos consistente do que o cultivo e aplicação de fósforo.

Termos para indexação: Brachiaria decumbens, Glycine max, adubo fosfatado, fungo micorrízico.

\section{Phosphorus fractions in Oxisols}

\begin{abstract}
The objective of this work was to verify if the historical use, phosphorus fertilization, mycorrhizal inoculation and cultivation with brachiaria and soybeans in greenhouse alter $\mathrm{P}$ fractions in three Oxisols. P linked to $\mathrm{Al}$ (P-Al), Fe (P-Fe), and $\mathrm{Ca}$ (P-Ca) were determined. The P fractions were separated with resin, $\mathrm{NaHCO}_{3}, \mathrm{NaOH}, \mathrm{HCl}$, and digestion with $\mathrm{H}_{2} \mathrm{SO}_{4}$ and $\mathrm{H}_{2} \mathrm{O}_{2}$. The organic and inorganic fractions were separated by autoclaving. In the cohesive Yellow Latosol (LAx) and acric Red Latosol (LVw), the P-Al, P-Fe, and P-Ca were higher in the cultivated soils, and in the dystroferric Red Latosol (LVdf), the P-Al and P-Fe were higher in the non-cultivated soils, trends non-altered by the cultivation with brachiaria and soybeans. $\mathrm{P}$ addition elevated mainly the labile inorganic (Pi-resin and $\mathrm{Pi}-\mathrm{NaHCO}_{3}$ ) and the moderately labile $(\mathrm{Pi}-\mathrm{NaOH})$ fractions. Soils formerly cultivated presents less $\mathrm{P}$ in the organic fractions than the non-cultivated ones. In soils with brachiaria, less $\mathrm{P}$ in the less labile fractions and more $\mathrm{P}$ in the more labile ones were verified. The effect of mycorrhizae was smaller and less consistent than the cultivation and $\mathrm{P}$ application.
\end{abstract}

Index terms: Brachiaria decumbens, Glycine max, phosphate fertilizers, mycorrhizal fungi.

(1) Aceito para publicação em 28 de julho de 2003.

Extraído da dissertação de mestrado apresentada pela primeira autora à Universidade Federal de Lavras (UFLA), Lavras, MG.

(2) UFLA, Dep. de Ciência do Solo, Caixa Postal 37, CEP 37200-000 Lavras, MG. E-mail: meire@hotmail.com, julionobrega@zipmail.com.br, siqueira@ufla.br,jmarques@ufla.br

(3) Embrapa-Centro Nacional de Pesquisa de Solos, Rua Jardim Botânico, 1024, CEP 22460-000 Rio de Janeiro, RJ. E-mail: motta@cnps.embrapa.br

\section{Introdução}

Para entender a condição e disponibilidade do P no solo, têm sido desenvolvidos métodos visando fracioná-lo de acordo com a disponibilidade, já que ocorre sob diferentes formas, caracterizadas por distintas capacidades de adsorção, principalmente em compostos de Fe, Al, Ca e matéria orgânica (Raij \& Feitosa, 1980). Entre os métodos de fracionamento, o mais conhecido é o de Chang \& Jackson (1957), 
que permite a caracterização, mediante extrações sucessivas, das diversas formas inorgânicas de P, baseada na capacidade de determinadas substâncias químicas em dissolver compostos específicos de $\mathrm{P}$ inorgânico do solo. Esse método, pela sua simplicidade, possibilidade de revelar a causa da deficiência desse elemento e prever a resposta das culturas à adubação fosfatada, tem sido muito utilizado nos estudos sobre o comportamento químico do P nos solos. Outro método bastante empregado é o de Hedley et al. (1982), que baseia-se na extração seqüencial de formas lábeis, pouco lábeis e não-lábeis, por meio da qual as formas inorgânicas e orgânicas são separadas conforme sua disponibilidade às plantas. Segundo Schmidt et al. (1997), a vantagem deste método é a obtenção de informações sobre a disponibilidade de $\mathrm{P}$ a curto e longo prazo, por meio dos valores das várias frações de $\mathrm{P}$, de vários graus de disponibilidade para as plantas, podendo também ser empregado para acompanhar modificações nas formas de P, em decorrência de processos no solo (Araújo \& Salcedo, 1997). O interesse na utilização deste método para o estudo da dinâmica do P em solos das regiões tropicais e subtropicais tem aumentado e envolve, especialmente, o uso de fertilizantes e o manejo do solo (Rheinheimer et al., 2000).

Em solos tropicais, qualquer tipo de manejo que aumente a absorção e utilização do P pelas plantas é importante para a agricultura. Uma alternativa proposta e estudada tem sido o emprego de micorrizas, entre outros fatores, pela exploração de maior volume do solo e capacidade das hifas em absorver P em baixas concentrações, favorecendo o crescimento das plantas em condições subótimas desse elemento (Siqueira, 1994). Em Latossolos, Alves (1988) verificou que estilosantes utilizam $\mathrm{P}-\mathrm{Al}$ e a braquiária, além do $\mathrm{P}-\mathrm{Al}$, o P-Fe no processo de absorção de fósforo. Segundo esse autor, plantas micorrizadas utilizam mais eficientemente o P menos disponível no solo, principalmente as formas ligadas a Fe e alumínio.

Embora essa simbiose exerça amplo efeito na nutrição fosfatada das plantas, a magnitude dos benefícios da micorrização depende de vários fatores, entre os quais os níveis de P no solo e a espécie de planta (Siqueira, 1994). Conhecer o efeito da aduba- ção fosfatada sobre as frações de P no solo, e outros fatores que influenciam essas frações no solo, à exemplo das micorrizas, ajudam na obtenção de informações importantes no manejo desse nutriente.

O objetivo deste trabalho foi verificar se o histórico de uso, adubação fosfatada, inoculação micorrízica e cultivo com braquiária e soja, em casa de vegetação, alteram as frações de $\mathrm{P}$ em três Latossolos.

\section{Material e Métodos}

Foram selecionados um Latossolo Amarelo coeso (LAx) textura argilosa, no Município de Linhares, ES, um Latossolo Vermelho ácrico $(\mathrm{LVw})$ textura muito argilosa, no Município de Uberlândia, MG, e um Latossolo Vermelho distroférrico (LVdf) textura muito argilosa, no Município de Patos de Minas, MG, todos coletados na camada de 0-20 cm (amostras compostas), sob condição de vegetação nativa (floresta tropical subperenifólia, cerrado tropical subcaducifólio e floresta tropical subcaducifólia, respectivamente) e sob cultivo convencional por mais de 10 anos, envolvendo calagens e adubações fosfatadas periódicas. O LAx sob cultivo recebeu por mais de 11 anos culturas anuais (feijão e milho), cujo solo, nos três anos precedentes à amostragem, permaneceu coberto com capim colonião (Panicum maximum). O LVw e LVdf cultivados também receberam culturas anuais por mais de 10 e 15 anos, respectivamente. Neste último, nos cinco anos precedentes à amostragem, o solo permaneceu coberto com capim braquiária (Brachiaria decumbens).

Após a coleta, as amostras compostas de solo foram secadas ao ar, destorroadas e passadas em peneira de $5 \mathrm{~mm}$ para os experimentos com plantas em casa de vegetação. Parte das amostras compostas foi passada em peneira de 2 mm e submetida a análises físicas e químicas. A análise física envolveu a determinação da composição granulométrica da TFSA, pelo método da pipeta (Day, 1965), com cinco replicatas. As análises químicas foram realizadas de acordo com Embrapa (1997) e incluíram pH, complexo sortivo e C orgânico, com três replicatas por determinação. A disponibilidade de $\mathrm{P}$ foi avaliada por Mehlich-1 (Embrapa, 1997) e resina de troca iônica (Raij \& Feitosa, 1980). Na Tabela 1 são apresentados os resultados da caracterização física e química dos solos estudados.

Dois experimentos foram realizados em casa de vegetação, um com braquiária e outro com soja (Glycine max) variedade CAC-1, em vasos com capacidade para $4 \mathrm{~kg}$ de solo. Os solos receberam calcário, na quantidade indicada para elevar o valor da saturação por bases a $60 \%$, e adubação básica combinada com a aplicação de duas doses de $\mathrm{P}$, 
que constituíram os tratamentos, definida com base em ensaio preliminar com milho, em casa de vegetação. Nas amostras destinadas ao cultivo com braquiária, aplicou-se $\mathrm{NH}_{4} \mathrm{NO}_{3}$ para atingir uma concentração no solo de $80 \mathrm{mg} \mathrm{kg}^{-1}$ de nitrogênio. Logo após, os solos foram incubados por 45 dias, mantendo-os com $60 \%$ do volume total de poros preenchidos com água. Em seguida, foram revolvidos e fumigados com brometo de metila. Após ventilação do material foi feita a inoculação de uma suspensão de esporos, obtida pelo método de Gerdemann \& Nicolson (1963), sendo o volume e concentração ajustados para aplicação de 300 esporos por vaso. A inoculação foi realizada em metade dos vasos utilizando-se $1 \mathrm{~mL}$ da suspensão de esporos, em orifícios feitos no solo. Em seguida, procedeuse a semeadura. No experimento com a braquiária, utilizou-se cerca de 10 sementes, e com a soja, quatro sementes por vaso, sendo mantidas, após o desbaste, duas plantas por vaso. Antes da semeadura, as sementes de soja foram submetidas à inoculação de Bradyrhizobium japonicum procedente da Embrapa-Centro Nacional de Pesquisa de Agrobiologia.

Nos dois experimentos, braquiária e soja, os tratamentos foram dispostos em delineamento inteiramente casualizado, em esquema fatorial 2x2x2 com três repetições, totalizando 144 vasos. Os tratamentos foram constituídos por duas condições de uso anterior do solo (solo nunca cultivado (NC) e solo cultivado (C) por vários anos); doses de P (P1 e P2), correspondentes a $5 \%$ e $50 \%$ da dose indicada para a produção máxima (determinada com base em experimento preliminar), de 15 e 150 (LAx), 20 e 200 (LVw) e 30 e $300 \mathrm{mg} \mathrm{kg}^{-1}$ (LVdf); e inoculação de fungo micorrízico Glomus etunicatum (inoculado e não-inoculado).

Após dois cultivos sucessivos com cada espécie em casa de vegetação, amostras de solo de cada tratamento e amostras coletadas no campo foram moídas em gral de ágata e passadas em peneiras de 100 meshes. Em seguida, foram determinadas as frações de P segundo os métodos de Chang \& Jackson (1957) e Hedley et al. (1982). No fracionamento de Chang \& Jackson (1957), foram separadas as formas de $\mathrm{P}$ ligadas a $\mathrm{Al}(\mathrm{P}-\mathrm{Al})$, extraídas com $\mathrm{NH}_{4} \mathrm{~F}$ 0,5 mol L-1 a pH 8,2; P ligado a Fe (P-Fe), extraído com $\mathrm{NaOH}$ 0,1 mol L-1; e P ligado a Ca (P-Ca), extraído com $\mathrm{H}_{2} \mathrm{SO}_{4} 0,25 \mathrm{~mol} \mathrm{~L}^{-1}$. Após a extração do P-Al, o solo residual foi centrifugado duas vezes com $\mathrm{NaCl} 1 \mathrm{~mol} \mathrm{~L}^{-1} \mathrm{e}$ descartou-se o sobrenadante. No fracionamento proposto por Hedley et al. (1982), o P inorgânico mais lábil foi extraído com resina de troca aniônica saturada com bicarbonato (Pi-Resina); o P inorgânico lábil ( $\left.\mathrm{Pi}-\mathrm{NaHCO}_{3}\right)$ e $\mathrm{P}$ orgânico ( $\left.\mathrm{Po}-\mathrm{NaHCO}_{3}\right)$, adsorvidos nas superfícies dos colóides, foram extraídos com $\mathrm{NaHCO}_{3} 0,5 \mathrm{~mol} \mathrm{~L}^{-1}$; o P

Tabela 1. Atributos físicos e químicos do Latossolo Amarelo coeso (LAx), Latossolo Vermelho ácrico (LVw) e Latossolo Vermelho distroférrico (LVdf) não cultivados (NC) e cultivados (C) antes dos experimentos em casa de vegetação.

\begin{tabular}{|c|c|c|c|c|c|c|}
\hline \multirow{2}{*}{ Atributos } & \multicolumn{2}{|c|}{ LAx } & \multicolumn{2}{|c|}{$\mathrm{LVw}$} & \multicolumn{2}{|c|}{ LVdf } \\
\hline & $\mathrm{NC}$ & $\mathrm{C}$ & NC & $\mathrm{C}$ & NC & $\mathrm{C}$ \\
\hline Areia grossa $\left(\mathrm{g} \mathrm{kg}^{-1}\right)$ & 370 & 330 & 110 & 160 & 100 & 110 \\
\hline Areia fina $\left(\mathrm{g} \mathrm{kg}^{-1}\right)$ & 100 & 130 & 100 & 110 & 80 & 60 \\
\hline Silte $\left(\mathrm{g} \mathrm{kg}^{-1}\right)$ & 90 & 110 & 120 & 100 & 240 & 230 \\
\hline Argila $\left(\mathrm{g} \mathrm{kg}^{-1}\right)$ & 440 & 430 & 670 & 630 & 580 & 600 \\
\hline pH em água & 5,7 & 5,9 & 4,9 & 5,8 & 4,9 & 5,2 \\
\hline $\mathrm{Al}^{3+}\left(\mathrm{cmol}_{\mathrm{C}} \mathrm{dm}^{-3}\right)$ & 0,0 & 0,0 & 0,9 & 0,0 & 0,8 & 0,2 \\
\hline $\mathrm{Ca}^{2+}\left(\mathrm{cmol}_{\mathrm{C}} \mathrm{dm}^{-3}\right)$ & 3,2 & 3,0 & 1,2 & 3,7 & 2,3 & 2,2 \\
\hline $\mathrm{Mg}^{2+}\left(\mathrm{cmol}_{\mathrm{c}} \mathrm{dm}^{-3}\right)$ & 0,8 & 1,2 & 0,1 & 0,2 & 0,9 & 1,1 \\
\hline $\mathrm{K}^{+}\left(\mathrm{mg} \mathrm{dm}^{-3}\right)$ & 90 & 73 & 36 & 59 & 64 & 166 \\
\hline $\mathrm{H}^{+}+\mathrm{Al}^{3+}\left(\mathrm{cmol}_{\mathrm{C}} \mathrm{dm}^{-3}\right)$ & 4,0 & 2,3 & 9,8 & 2,9 & 12,3 & 7,0 \\
\hline P-Mehlich-1 (mg dm ${ }^{-3}$ ) & 4 & 22 & 2 & 45 & 78 & 63 \\
\hline P-resina ( $\mathrm{mg} \mathrm{dm}^{-3}$ ) & 11 & 23 & 7 & 48 & 91 & 57 \\
\hline $\mathrm{S}\left(\mathrm{cmol}_{\mathrm{C}} \mathrm{dm}^{-3}\right)$ & 4,2 & 4,4 & 1,4 & 4,1 & 3,4 & 3,7 \\
\hline $\mathrm{t}\left(\mathrm{cmol}_{\mathrm{C}} \mathrm{dm}^{-3}\right)$ & 4,2 & 4,4 & 2,3 & 4,1 & 4,2 & 3,9 \\
\hline $\mathrm{T}\left(\mathrm{cmol}_{\mathrm{c}} \mathrm{dm}^{-3}\right)$ & 8,2 & 6,7 & 11,2 & 7,0 & 15,7 & 10,7 \\
\hline V (\%) & 51 & 66 & 13 & 58 & 21 & 35 \\
\hline m (\%) & 0 & 0 & 39 & 0 & 19 & 5 \\
\hline C org. $\left(\mathrm{g} \mathrm{kg}^{-1}\right)$ & 22 & 14 & 26 & 19 & 50 & 32 \\
\hline $\mathrm{P}-\mathrm{H}_{2} \mathrm{SO}_{4}\left(\mathrm{mg} \mathrm{kg}^{-1}\right)$ & 30,6 & 30,6 & 148,5 & 200,9 & $3.624,4$ & $4.528,4$ \\
\hline
\end{tabular}


inorgânico (Pi-NaOH) associado aos óxidos de Fe e Al e o P orgânico (Po-NaOH), associado aos compostos húmicos, foram extraídos com $\mathrm{NaOH} 0,5 \mathrm{~mol} \mathrm{~L}^{-1}$; o P ligado a Ca, considerado não-disponível a curto prazo, foi extraído com $\mathrm{HCl} 1 \mathrm{~mol} \mathrm{~L}^{-1}$; e finalmente no resíduo, o P inorgânico e P orgânico, mais quimicamente estáveis, foram determinados por digestão usando $\mathrm{H}_{2} \mathrm{SO}_{4}+\mathrm{H}_{2} \mathrm{O}_{2}$ concentrados. Nos extratos do bicarbonato, bicarbonato após tratamento com clorofórmio, extrato acidificado do bicarbonato e hidróxido de Na procedeu-se a determinação do $\mathrm{P}$ com e sem autoclavagem, para a separação do $\mathrm{P}$ inorgânico e P orgânico. O P nos extratos foi determinado por colorimetria, usando o método de Murphy \& Riley (1962). Na Tabela 2 são apresentados os resultados da caracterização de P, com ambos os métodos, antes dos cultivos em casa de vegetação.

Os dados obtidos foram submetidos à análise de variância e as médias relativas aos dados de fracionamento de P foram comparadas pelo teste de Tukey a 5\% de probabilidade, usando o programa de análises estatísticas SISVAR.

\section{Resultados e Discussão}

Os resultados do fracionamento de $\mathrm{P}$, com ambos os métodos e culturas, foram apresentados somente quando significativos. As interações duplas (Tabelas 3, 4 e 5) foram desdobradas de forma a verificar os seguintes efeitos: doses de P dentro de cada histórico de uso; inoculação dentro de cada histórico de uso; e inoculação dentro de cada dose de fósforo. A interação tripla, observada em algumas formas de P nos solos com braquiária e soja, não foi discutida neste trabalho.

No fracionamento de Chang \& Jackson (1957) nos solos com braquiária, verificou-se na fração $\mathrm{P}-\mathrm{Al}$ efeito individual da adubação no $\mathrm{LVdf}(\mathrm{P} 1<\mathrm{P} 2)$. Nos demais solos, o efeito do $\mathrm{P}$ ocorreu nas interações LAx (P x I) e LVw (H x P) (Tabela 3). No LAx, o tratamento P2 aumentou a fração somente na ausência da inoculação, o que mostra o efeito das micorrizas na utilização dessa fração, conforme também verificado por Alves (1988). Também foram verificados efeitos individuais do histórico no LAx $(\mathrm{NC}<\mathrm{C})$ e LVdf $(\mathrm{NC}>\mathrm{C})$.

A fração P-Fe foi influenciada pela aplicação de P, principalmente no LVdf, no qual ocorreu efeito individual $(\mathrm{P} 1<\mathrm{P} 2)$. No LAx e no LVw o efeito do $\mathrm{P}$ ocorreu na interação H x P. No LVw foi verificado o efeito da interação H x I. A inoculação aumentou essa fração no solo não cultivado.

Na fração P-Ca, verificou-se efeito individual do histórico no LAx $(\mathrm{NC}<\mathrm{C})$, no LVw $(\mathrm{NC}<\mathrm{C})$ e no LVdf $(\mathrm{NC}<\mathrm{C})$. Maior quantidade de $\mathrm{P}-\mathrm{Ca}$ em solos cultivados pode ser atribuída à atividade do Ca proporci-

Tabela 2. Fracionamento de $\mathrm{P}\left(\mathrm{mg} \mathrm{kg}^{-1}\right)$ de amostras de solo coletadas no campo antes dos experimentos em casa de vegetação, segundo os métodos de Chang \& Jackson (1957) e Hedley et al. (1982), em três Latossolos previamente cultivados (C) e não cultivados (NC) $)^{(1)}$.

\begin{tabular}{|c|c|c|c|c|c|c|}
\hline \multirow[t]{2}{*}{ Fracionamento } & \multicolumn{2}{|c|}{ LAx } & \multicolumn{2}{|c|}{$\mathrm{LVw}$} & \multicolumn{2}{|c|}{ LVdf } \\
\hline & $\mathrm{NC}$ & $\mathrm{C}$ & $\mathrm{NC}$ & $\mathrm{C}$ & $\mathrm{NC}$ & $\mathrm{C}$ \\
\hline & \multicolumn{6}{|c|}{ Chang \& Jackson (1957) } \\
\hline $\mathrm{P}-\mathrm{Al}$ & 22 & 50 & 23 & 79 & 703 & 504 \\
\hline $\mathrm{P}-\mathrm{Fe}$ & 10 & 39 & 35 & 85 & 839 & 839 \\
\hline \multirow[t]{2}{*}{$\mathrm{P}-\mathrm{Ca}$} & 1 & 2 & 5 & 33 & 283 & 341 \\
\hline & \multicolumn{6}{|c|}{ Hedley et al. (1982) } \\
\hline P-resina & 6 & 13 & 4 & 30 & 129 & 86 \\
\hline Pi-bicarbonato & 2 & 14 & 6 & 18 & 153 & 105 \\
\hline Po-bicarbonato & 17 & 20 & 18 & 15 & 59 & 23 \\
\hline $\mathrm{Pi}-\mathrm{NaOH}$ & 24 & 65 & 63 & 145 & 1.707 & 1.615 \\
\hline $\mathrm{Po}-\mathrm{NaOH}$ & 47 & 29 & 71 & 62 & 327 & 279 \\
\hline $\mathrm{P}-\mathrm{HCl}$ & 2 & 14 & 1 & 41 & 150 & 135 \\
\hline P-residual & 650 & 969 & 1.162 & 1.320 & 2.581 & 4.113 \\
\hline
\end{tabular}

(1)LAx: Latossolo Amarelo coeso; LVw: Latossolo Vermelho ácrico; LVdf: Latossolo Vermelho distroférrico. 
onada pela calagem em cultivos anteriores ao realizado em casa de vegetação e por fertilizantes fosfatados, conforme observado por Novelino (1999). No LAx também foi verificado o efeito da interação P x I com aumento da fração por P2 somente na ausência da inoculação.

Nos solos com soja verificou-se, em relação ao $\mathrm{P}-\mathrm{Al}$, efeito individual da adubação no LVdf $(\mathrm{P} 1<\mathrm{P} 2)$, o que também ocorreu no solo com braquiária. No LAx e LVw, o efeito do P ocorreu na interação H x P. Observaram-se também efeito individual da inoculação no LAx (NI>I) e efeito da interação H x I no LVdf

A fração P-Fe foi influenciada pela aplicação de P nos três solos, sendo no LVdf individualmente
$(\mathrm{P} 1<\mathrm{P} 2)$, também verificado na braquiária. No LAx o efeito do $\mathrm{P}$ depende do histórico $(\mathrm{H} \times \mathrm{P})$ e da inoculação (P x I). Neste caso, o aumento da fração por P2 ocorreu somente quando a soja não foi inoculada. No LVw o efeito do P foi influenciado pelo histórico $(\mathrm{H} \times \mathrm{P})$.

Na fração P-Ca, o efeito do P ocorreu somente na interação P x I, no LVdf, com aumento desta fração pela inoculação na dose P1. No LVdf observou-se também efeito individual do histórico $(\mathrm{NC}<\mathrm{C})$. No $\mathrm{LVw}$ foi verificado efeito da interação $\mathrm{H}$ x I, com redução do P-Ca pela inoculação no solo cultivado.

No geral, independentemente das culturas, a fração P-Fe predominou no LVw e no LVdf, indicando um maior grau de solubilização das formas $\mathrm{P}-\mathrm{Al}$ e

Tabela 3. P-Al, P-Fe e P-Ca $\left(\mathrm{mg} \mathrm{kg}^{-1}\right)$ nos solos cultivados com braquiária e soja em resposta à aplicação de duas doses de P (P1 e P2) e inoculação (I) ou não (NI) de fungo micorrízico em três Latossolos previamente cultivados (C) e não cultivados (NC) $)^{(1)}$.

\begin{tabular}{|c|c|c|c|c|c|c|c|c|c|c|}
\hline \multirow{3}{*}{$\begin{array}{l}\text { Histórico e } \\
\text { fósforo }\end{array}$} & \multicolumn{4}{|c|}{ LAx } & \multicolumn{4}{|c|}{$\mathrm{LVw}$} & \multirow{2}{*}{\multicolumn{2}{|c|}{$\begin{array}{l}\text { LVdf } \\
\text { Micorriza }\end{array}$}} \\
\hline & \multicolumn{2}{|c|}{ Fósforo } & \multicolumn{2}{|c|}{ Micorriza } & \multicolumn{2}{|c|}{ Fósforo } & \multicolumn{2}{|c|}{ Micorriza } & & \\
\hline & P1 & P2 & NI & I & P1 & $\mathrm{P} 2$ & NI & I & NI & I \\
\hline & \multirow{2}{*}{\multicolumn{10}{|c|}{$\begin{array}{l}\text { Solos cultivados com braquiária } \\
\text { P-Al (mg kg-1) }\end{array}$}} \\
\hline & & & & & & & & & & \\
\hline NC & -(2) & - & - & - & $17 \mathrm{~b}$ & $78 a$ & - & - & - & - \\
\hline $\mathrm{C}$ & - & - & - & - & $72 b$ & $159 a$ & - & - & - & - \\
\hline $\mathrm{P} 1$ & - & - & $23 a$ & $26 a$ & - & - & - & - & - & - \\
\hline \multirow[t]{2}{*}{$\mathrm{P} 2$} & - & - & 103a & $97 \mathrm{~b}$ & - & - & - & - & - & - \\
\hline & \multicolumn{10}{|c|}{ P-Fe $\left(\mathrm{mg} \mathrm{kg}^{-1}\right)$} \\
\hline NC & $7 \mathrm{~b}$ & $13 a$ & - & - & $45 b$ & $106 \mathrm{a}$ & $2 b$ & 78a & - & - \\
\hline \multirow[t]{2}{*}{$\mathrm{C}$} & $30 \mathrm{~b}$ & $53 a$ & - & - & $108 \mathrm{~b}$ & $153 a$ & 133a & $128 \mathrm{a}$ & - & - \\
\hline & \multicolumn{10}{|c|}{ P-Ca $\left(\mathrm{mg} \mathrm{kg}^{-1}\right)$} \\
\hline $\mathrm{P} 1$ & - & - & $2 \mathrm{a}$ & $2 \mathrm{a}$ & - & - & - & - & - & - \\
\hline \multirow[t]{2}{*}{$\mathrm{P} 2$} & - & - & 3a & $1 \mathrm{~b}$ & - & - & - & - & - & - \\
\hline & \multicolumn{10}{|c|}{$\begin{array}{l}\text { Solos cultivados com soja } \\
\text { P-Al }\left(\mathrm{mg} \mathrm{kg}^{-1}\right)\end{array}$} \\
\hline NC & $21 b$ & $86 a$ & - & - & $23 b$ & $96 a$ & - & - & $852 a$ & $798 b$ \\
\hline \multirow[t]{2}{*}{$\mathrm{C}$} & $35 b$ & $91 \mathrm{a}$ & - & - & $83 b$ & $188 \mathrm{a}$ & - & - & $592 b$ & $615 a$ \\
\hline & \multicolumn{10}{|c|}{ P-Fe $\left(\mathrm{mg} \mathrm{kg}^{-1}\right)$} \\
\hline NC & $11 b$ & $22 \mathrm{a}$ & - & - & $44 \mathrm{~b}$ & $102 a$ & - & - & - & - \\
\hline $\mathrm{C}$ & $43 \mathrm{~b}$ & $61 \mathrm{a}$ & - & - & $101 b$ & 148a & - & - & - & - \\
\hline $\mathrm{P} 1$ & - & - & $26 a$ & $27 a$ & - & - & - & - & - & - \\
\hline \multirow[t]{2}{*}{$\mathrm{P} 2$} & - & - & 43a & $40 \mathrm{~b}$ & - & - & - & - & - & - \\
\hline & \multicolumn{10}{|c|}{ P-Сa (mg kg $\left.{ }^{-1}\right)$} \\
\hline $\mathrm{NC}$ & - & - & - & - & - & - & $2 \mathrm{a}$ & $1 \mathrm{a}$ & - & - \\
\hline $\mathrm{C}$ & - & - & - & - & - & - & $29 a$ & $23 b$ & - & - \\
\hline P1 & - & - & - & - & - & - & - & - & $286 b$ & $297 a$ \\
\hline P2 & - & - & - & - & - & - & - & - & $312 a$ & $307 a$ \\
\hline
\end{tabular}

(1)LAx: Latossolo Amarelo coeso; LVw: Latossolo Vermelho ácrico; LVdf: Latossolo Vermelho distroférrico. (2)Interação não-significativa. 
retenção como P-Fe, o que, segundo Novelino (1999), tem sido comum, especialmente em solos intemperizados, com o aumento do tempo de contato entre fosfato e solo. A fração P-Ca foi menor que as frações P-Fe e P-Al, característica, segundo Bahia Filho \& Braga (1975), de solos mais intemperizados, pobres em Ca, pois à medida que vão se tornando mais ácidos, pela remoção de sílica e bases, formas mais solúveis de P-Ca são convertidas em P-Al e P-Fe (Hsu, 1977).

O efeito das micorrizas no aumento da disponibilidade de $\mathrm{P}$ às plantas, oriundo das diferentes frações, foi menos evidente e, da mesma forma como o histórico e a aplicação de $\mathrm{P}$, influenciou de maneira diferenciada as frações de P nos solos. Nos solos com soja, o efeito da inoculação no aumento da absorção das formas de $\mathrm{P}$ foi mais evidente do que nos solos com braquiária, já que ocorreu tanto de forma individual quanto em interação numa proporção maior sobre as diferentes formas de P, mostrando ser a interação soja-micorriza mais eficiente na utilização do $\mathrm{P}$ ligado às frações do que a interação braquiária-micorriza.

No fracionamento de Hedley et al. (1982), nos solos com braquiária (Tabela 4), na fração Pi-resina, forma mais disponível às plantas, verificou-se efeito do $\mathrm{P}$ em todos os solos, principalmente no LVdf $(\mathrm{P} 1<\mathrm{P} 2)$. No LAx e LVw, o efeito do P foi influenciado pelo histórico ( $\mathrm{H}$ x P).

A aplicação de $\mathrm{P}$ aumentou o teor de $\mathrm{Pi}-\mathrm{NaHCO}_{3}$, fração inorgânica lábil, em todos os solos, principalmente no LVdf, onde ocorreu de forma individual

Tabela 4. P-resina, Pi-NaHCO 3 , Po- $\mathrm{NaHCO}_{3}$, Pi-NaOH, Po-NaOH, P-HCl e P-residual (mg kg-1) nos solos cultivados com braquiária em resposta à aplicação de duas doses de P (P1 e P2) e inoculação (I) ou não (NI) de fungo micorrízico em três Latossolos previamente cultivados (C) e não cultivados (NC) ${ }^{(1)}$.

\begin{tabular}{|c|c|c|c|c|c|c|c|c|c|c|}
\hline \multirow{3}{*}{$\begin{array}{c}\text { Histórico } \\
\text { e } \\
\text { fósforo }\end{array}$} & \multicolumn{4}{|c|}{ LAx } & \multicolumn{4}{|c|}{$\mathrm{LVw}$} & \multirow{2}{*}{\multicolumn{2}{|c|}{$\begin{array}{c}\text { LVdf } \\
\text { Fósforo }\end{array}$}} \\
\hline & \multicolumn{2}{|c|}{ Fósforo } & \multicolumn{2}{|c|}{ Micorriza } & \multicolumn{2}{|c|}{ Fósforo } & \multicolumn{2}{|c|}{ Micorriza } & & \\
\hline & P1 & P2 & $\mathrm{NI}$ & I & P1 & P2 & $\mathrm{NI}$ & $\mathrm{I}$ & $\mathrm{P} 1$ & $\mathrm{P} 2$ \\
\hline & \multicolumn{10}{|c|}{ P-Resina (mg kg-1) } \\
\hline $\mathrm{NC}$ & $2 b$ & $18 \mathrm{a}$ & $-^{(2)}$ & - & 3b & $14 \mathrm{a}$ & - & - & - & - \\
\hline \multirow[t]{2}{*}{$\mathrm{C}$} & $7 \mathrm{~b}$ & 29a & - & - & $32 b$ & $50 \mathrm{a}$ & - & - & - & - \\
\hline & \multicolumn{10}{|c|}{$\mathrm{Pi}-\mathrm{NaHCO}_{3}\left(\mathrm{mg} \mathrm{kg}^{-1}\right)$} \\
\hline $\mathrm{NC}$ & $3 b$ & $7 a$ & $6 a$ & $4 \mathrm{~b}$ & $4 b$ & $22 \mathrm{a}$ & - & - & - & - \\
\hline $\mathrm{C}$ & $10 \mathrm{~b}$ & $29 a$ & $19 a$ & $20 a$ & $12 b$ & $18 a$ & - & - & - & - \\
\hline $\mathrm{P} 1$ & - & - & $6 a$ & $7 \mathrm{a}$ & - & - & - & - & - & - \\
\hline \multirow[t]{2}{*}{$\mathrm{P} 2$} & - & - & $19 a$ & $17 \mathrm{~b}$ & - & - & - & - & - & - \\
\hline & \multicolumn{10}{|c|}{$\mathrm{Po}-\mathrm{NaHCO}_{3}\left(\mathrm{mg} \mathrm{kg}^{-1}\right)$} \\
\hline $\mathrm{NC}$ & $12 b$ & $27 a$ & - & - & $14 \mathrm{a}$ & $15 a$ & - & - & $138 b$ & $171 \mathrm{a}$ \\
\hline \multirow[t]{2}{*}{$\mathrm{C}$} & $10 \mathrm{a}$ & $12 \mathrm{a}$ & - & - & $14 \mathrm{~b}$ & $28 a$ & - & - & $115 a$ & $124 \mathrm{a}$ \\
\hline & \multicolumn{10}{|c|}{$\mathrm{Pi}-\mathrm{NaOH}\left(\mathrm{mg} \mathrm{kg}^{-1}\right)$} \\
\hline $\mathrm{NC}$ & $9 b$ & $26 a$ & - & - & $30 b$ & $53 a$ & - & - & - & - \\
\hline $\mathrm{C}$ & $24 b$ & $46 a$ & - & - & $103 b$ & $135 a$ & - & - & - & - \\
\hline $\mathrm{P} 1$ & - & - & $18 a$ & $15 b$ & - & - & $68 a$ & $66 a$ & - & - \\
\hline \multirow[t]{2}{*}{$\mathrm{P} 2$} & - & - & $38 a$ & $35 b$ & - & - & $93 b$ & $96 a$ & - & - \\
\hline & \multicolumn{10}{|c|}{ Po-NaOH (mg kg-1 $)$} \\
\hline $\mathrm{NC}$ & - & - & - & - & $72 b$ & $132 \mathrm{a}$ & - & - & - & - \\
\hline $\mathrm{C}$ & - & - & - & - & $73 b$ & $96 a$ & - & - & - & - \\
\hline P1 & - & - & $23 a$ & $20 b$ & - & - & - & - & - & - \\
\hline \multirow[t]{2}{*}{$\mathrm{P} 2$} & - & - & $34 a$ & $35 a$ & - & - & - & - & - & - \\
\hline & \multicolumn{10}{|c|}{$\mathrm{P}-\mathrm{HCl}\left(\mathrm{mg} \mathrm{kg}^{-1}\right)$} \\
\hline $\mathrm{NC}$ & - & - & - & - & $2 \mathrm{a}$ & $3 a$ & - & - & - & - \\
\hline $\mathrm{C}$ & - & - & - & - & $41 b$ & $52 a$ & - & - & - & - \\
\hline
\end{tabular}

${ }^{(1)}$ LAx: Latossolo Amarelo coeso; LVw: Latossolo Vermelho ácrico; LVdf: Latossolo Vermelho distroférrico. (2)Interação não-significativa. 
(P1<P2). Aumento no P lábil com a aplicação de fertilizantes foi verificado por Duffera \& Robarge (1996). Nos demais solos, o efeito do $\mathrm{P}$ ocorreu em interação LAx (H x P) e mostrou que o aumento com a dose P2 ocorre principalmente no solo cultivado, já que a inoculação no solo não cultivado (H x I) reduziu a fração quando a dose $\mathrm{P} 2$ foi adicionada ao solo (P x I). No LVw foi verificado somente o efeito da interação $\mathrm{H}$ x P, e no LVdf, efeito individual de histórico (NC>C).

As frações inorgânicas lábeis, Pi-resina e $\mathrm{Pi}-\mathrm{NaHCO}_{3}$ constituíram as menores frações nos solos deste estudo - tendência esperada em solos altamente intemperizados (Wright \& Coleman, 1999).

Na fração Po- $\mathrm{NaHCO}_{3}$, mais lábil entre as orgânicas, o efeito da adubação ocorreu apenas na interação H x P, com aumento dessa fração no solo não cultivado do LAx e LVdf e no cultivado do LVw. No LVw não cultivado pode ter ocorrido maior mineralização de Po com P2. Perdas ou ganhos de Po com a adição de $\mathrm{P}$ foram também observados por Araújo \& Salcedo (1997).

A fração $\mathrm{Pi}-\mathrm{NaOH}$, considerada pouco disponível às plantas (Tiessen et al., 1984), foi a fração inorgânica predominante (maior que P-resina e $\mathrm{P}-\mathrm{NaHCO}_{3}$ ), concordando com o trabalho de Cross \& Schlesinger (1995). A aplicação de P aumentou a fração $\mathrm{Pi}-\mathrm{NaOH}$ em todos os solos, principalmente no LVdf $(\mathrm{P} 1<\mathrm{P} 2)$. Nos demais solos, o efeito do $\mathrm{P}$ ocorreu nas interações H x P e P x I. No LAx, independentemente da dose, a inoculação reduziu a fração; no LVw, houve ligeiro aumento com P2.

A fração Po-NaOH foi maior que a $\mathrm{Po}-\mathrm{NaHCO}_{3}$, concordando com Cassagne \& Remaury (2000). Na fração Po-NaOH o efeito da adubação ocorreu apenas na interação P x I no solo Lax, com redução da fração pela inoculação somente na dose P1, e na interação H x P no solo LVw. Aumento do P orgânico pela fertilização foi verificado por Tiessen et al. (1992). No LAx e no LVdf foram verificados também efeitos individuais do histórico ( $\mathrm{NC}<\mathrm{C}$ e $\mathrm{NC}>\mathrm{C}$, respectivamente). Araújo et al. (1993) obtiveram maior Po-NaOH em área cultivada do que em área sob vegetação nativa.

A fração P-HCl é associada principalmente com compostos insolúveis de $\mathrm{P}-\mathrm{Ca}$, tais como hidroxiapatita (Williams et al., 1980), sendo considerada não lábil. Na fração P-HCl, o efeito da adição de
$\mathrm{P}$ foi relativamente pequeno, à exceção no LAx, em que teve efeito individual $(\mathrm{P} 1<\mathrm{P} 2)$. No $\mathrm{LVw}$ o efeito do $\mathrm{P}$ ocorreu na interação $\mathrm{H} \times \mathrm{P}$, com aumento somente no solo cultivado. Baixos teores de $\mathrm{P}-\mathrm{HCl}$ são esperados, pois se trata de solos ácidos, porém, no LVw, o cultivo com adubações pesadas de P influenciou significativamente essa fração, provavelmente pela quantidade de Ca introduzida ao longo do período anterior de cultivo.

Das frações de P estudadas, a maior foi o P residual, composta pela fração húmus mais estável (Stewart et al., 1980) e formas inorgânicas muito insolúveis, consideradas muito resistentes à decomposição (Daroub et al., 2000). Nessa fração ocorreu efeito individual do histórico nos três solos ( $\mathrm{NC}<\mathrm{C}$ ). Aumentos nos solos cultivados são provavelmente decorrentes de adubações recebidas anteriormente, que contribuíram para a transformação do fertilizante fosfatado em formas menos disponíveis de $\mathrm{P}$ (aumento da fixação) a longo prazo. Ausência do efeito do P aplicado como tratamento nesta fração foi verificada também por Beck \& Sanchez (1994).

Nos solos com soja (Tabela 5) verificou-se em relação ao P-resina que o efeito da adubação ocorre somente na interação com o histórico ( $\mathrm{H} \mathrm{x} \mathrm{P)} \mathrm{nos}$ três solos, e na interação com a inoculação (P x I) no LAx. Aumento da fração pela aplicação de P também foi verificado por Beck (1991) em um solo da Amazônia. Na interação P x I, nos solos LAx e LVdf, a inoculação reduziu a fração somente na dose P1. Segundo Saggin-Júnior et al. (1994), o efeito da micorrização diminui com a elevação do teor de P no solo. No LVdf foi verificado também o efeito da interação H x I.

$\mathrm{Na}$ fração $\mathrm{Pi}-\mathrm{NaHCO}_{3}$ foi verificado efeito da adubação fosfatada em todos os solos, principalmente no LVdf $\mathrm{P} 1<\mathrm{P} 2$, conforme também observado por Beck \& Sanchez (1994). No LAx e LVw o efeito do P ocorreu somente na interação $\mathrm{H}$ x P. No LVw, diferente do que ocorreu no cultivo com braquiária (Tabela 4), houve efeito individual da inoculação (NI>I), e na LVdf, efeito do histórico (NC>C) (Tabela 4).

$\mathrm{Na}$ fração $\mathrm{Po}-\mathrm{NaHCO}_{3}$ houve efeito da adubação somente em interação com o histórico (LAx, LVw e LVdf) e com a inoculação (LVdf). Tiessen et al. (1992) observaram manutenção ou aumento de Po em resposta à aplicação de fósforo. Na interação $\mathrm{P}$ x I, a 
inoculação aumentou a fração na dose P1, no solo não cultivado.

Na fração Pi-NaOH observou-se que o efeito da aplicação de P ocorreu somente na interação com o histórico (LAx, LVw e LVdf) e com a inoculação (LAx). Pelo efeito da interação P x I, a inoculação reduziu a fração na dose P1 e aumentou na dose P2. No LVw, o efeito da inoculação foi mais expressivo, pois ocorreu individualmente (NI>I), não sendo observado no solo com braquiária (Tabela 4). Portanto, sob menor disponibilidade de $\mathrm{P}$, a micorrização pode ter favorecido a soja na absorção do P ligado a essa fração.

O Po-NaOH foi a fração predominante do P orgânico extraível, conforme observado também na braquiária. A aplicação de P influenciou essa fração somente no LAx e no LVw pela interação $\mathrm{H}$ x P.
No LAx, foi observado também o efeito da interação H x I, com redução da fração pela inoculação no solo não cultivado. No LVdf, foi verificado o efeito do histórico (NC>C), também observado no solo com braquiária (Tabela 4). As frações de P orgânico foram variáveis, mas geralmente o cultivo reduziu os valores dessa fração no solo (Tabela 2), fato associado às reduções nos teores de $\mathrm{C}$ orgânico, sempre inferiores nos solos cultivados (Tabela 1), provavelmente por causa da maior mineralização da matéria orgânica provocada pelo cultivo.

Na fração P-HCl verificou-se que o efeito da adubação ocorreu somente no LVw, na interação com o histórico, com aumento da fração no solo cultivado. Efeito individual do histórico foi observado no LVdf $(\mathrm{NC}<\mathrm{C})$.

Tabela 5. P-resina, Pi-NaHCO 3 , Po- $\mathrm{NaHCO}_{3}$, Pi-NaOH, Po-NaOH, P-HCl e P-residual (mg kg-1) nos solos cultivados com soja em resposta à aplicação de duas doses de P (P1 e P2) e inoculação (I) ou não (NI) de fungo micorrízico em três Latossolos previamente cultivados (C) e não cultivados (NC) ${ }^{(1)}$.

\begin{tabular}{|c|c|c|c|c|c|c|c|c|c|c|}
\hline \multirow{3}{*}{$\begin{array}{c}\text { Histórico } \\
\text { e } \\
\text { fósforo }\end{array}$} & \multicolumn{4}{|c|}{ LAx } & \multirow{2}{*}{\multicolumn{2}{|c|}{$\begin{array}{c}\text { LVw } \\
\text { Fósforo }\end{array}$}} & \multicolumn{4}{|c|}{ LVdf } \\
\hline & \multicolumn{2}{|c|}{ Fósforo } & \multicolumn{2}{|c|}{ Micorriza } & & & \multicolumn{2}{|c|}{ Fósforo } & \multicolumn{2}{|c|}{ Micorriza } \\
\hline & $\mathrm{P} 1$ & $\mathrm{P} 2$ & NI & I & P1 & P2 & P1 & $\mathrm{P} 2$ & NI & I \\
\hline & \multicolumn{10}{|c|}{ P-Resina (mg kg-1) } \\
\hline $\mathrm{NC}$ & $4 \mathrm{~b}$ & $17 \mathrm{a}$ & $-(2)$ & - & $4 \mathrm{~b}$ & $13 a$ & $45 b$ & $53 a$ & $52 a$ & $46 b$ \\
\hline $\mathrm{C}$ & $11 b$ & $28 \mathrm{a}$ & - & - & I5b & $2 a$ & $34 b$ & $52 \mathrm{a}$ & $41 b$ & $46 a$ \\
\hline P1 & - & - & $8 a$ & $6 b$ & - & - & - & - & - & - \\
\hline \multirow[t]{2}{*}{$\mathrm{P} 2$} & - & - & $22 \mathrm{a}$ & $23 a$ & - & - & - & - & - & - \\
\hline & \multicolumn{10}{|c|}{$\mathrm{Pi}-\mathrm{NaHCO}_{3}\left(\mathrm{mg} \mathrm{kg}^{-1}\right)$} \\
\hline $\mathrm{NC}$ & $6 b$ & $18 \mathrm{a}$ & - & - & $13 a$ & $14 \mathrm{a}$ & - & - & - & - \\
\hline \multirow[t]{2}{*}{$\mathrm{C}$} & $13 \mathrm{~b}$ & $27 \mathrm{a}$ & - & - & $10 \mathrm{~b}$ & $29 a$ & - & - & - & - \\
\hline & \multicolumn{10}{|c|}{ 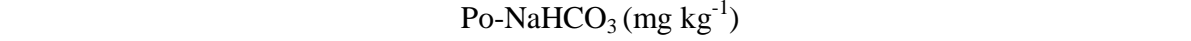 } \\
\hline $\mathrm{NC}$ & $16 b$ & $47 \mathrm{a}$ & - & - & $7 \mathrm{~b}$ & $25 \mathrm{a}$ & $70 \mathrm{~b}$ & $110 \mathrm{a}$ & $87 b$ & 93a \\
\hline $\mathrm{C}$ & $25 b$ & $48 a$ & - & - & $14 b$ & $25 a$ & 41b & $64 a$ & $54 a$ & $51 a$ \\
\hline P1 & - & - & - & - & - & - & - & - & $52 b$ & $59 a$ \\
\hline \multirow[t]{2}{*}{$\mathrm{P} 2$} & - & - & - & - & - & - & - & - & $89 a$ & $85 a$ \\
\hline & \multicolumn{10}{|c|}{$\mathrm{Pi}-\mathrm{NaOH}\left(\mathrm{mg} \mathrm{kg}^{-1}\right)$} \\
\hline $\mathrm{NC}$ & $22 b$ & $46 a$ & - & - & 43b & $74 a$ & $1.584 b$ & $1.835 a$ & - & - \\
\hline C & $53 \mathrm{~b}$ & $74 a$ & - & - & 146b & 200a & $1.441 \mathrm{~b}$ & $1.569 \mathrm{a}$ & - & - \\
\hline P1 & - & - & $39 a$ & $35 b$ & - & - & - & - & - & - \\
\hline \multirow[t]{2}{*}{$\mathrm{P} 2$} & - & - & $58 \mathrm{~b}$ & $61 \mathrm{a}$ & - & - & - & - & - & - \\
\hline & \multicolumn{10}{|c|}{ Po-NaOH (mg kg-1 $)$} \\
\hline $\mathrm{NC}$ & $45 b$ & $59 a$ & $54 b$ & $51 \mathrm{a}$ & $73 b$ & $88 a$ & - & - & - & - \\
\hline \multirow[t]{2}{*}{$\mathrm{C}$} & $32 b$ & $62 \mathrm{a}$ & $46 \mathrm{a}$ & $48 \mathrm{a}$ & $63 a$ & $64 a$ & - & - & - & - \\
\hline & \multicolumn{10}{|c|}{ P-HCl (mg kg-1) } \\
\hline $\mathrm{NC}$ & - & - & - & - & $2 \mathrm{a}$ & $2 \mathrm{a}$ & - & - & - & - \\
\hline $\mathrm{C}$ & - & - & - & - & $47 \mathrm{~b}$ & $73 a$ & - & - & - & - \\
\hline
\end{tabular}

${ }^{(1)}$ LAx: Latossolo Amarelo coeso; LVw: Latossolo Vermelho ácrico; LVdf: Latossolo Vermelho distroférrico. (2)Interação não-significativa. 
O P-residual foi a fração predominante, e conforme observado na braquiária (Tabela 4), foi influenciada somente pelo histórico nos três solos ( $\mathrm{NC}<\mathrm{C}$ ). Segundo Lilienfein et al. (2000), em sistemas cultivados, a fração de $\mathrm{P}$ inorgânico lábil aumenta e transforma-se lentamente em formas menos disponíveis com o tempo.

Conforme também verificado no fracionamento de Chang \& Jackson (1957), o cultivo e adubação fosfatada afetaram a distribuição do P nas diferentes frações de forma variável, conforme o tipo de cultura. No geral, o cultivo aumentou as frações inorgânicas (P-resina e $\mathrm{P}-\mathrm{HCl}$ ) e orgânicas (Po-NaHCO ${ }_{3}$ e Po- $\mathrm{NaOH}$ ), principalmente quando em interação com a aplicação de P, com aumento dessas frações na dose P2 nos solos não cultivados. O efeito da aplicação de P no aumento das frações inorgânicas lábeis e moderadamente lábeis de $\mathrm{P}$, principalmente nos solos não cultivados, ocorre, provavelmente, por causa da menor disponibilidade de $\mathrm{P}$ nesses solos. No LVdf, o efeito da aplicação de P ocorreu somente nas frações lábeis que tiveram os teores de P reduzidos pelo cultivo. As frações não lábeis beneficiaram-se muito pouco da aplicação de fósforo.

No geral, a inoculação contribuiu para aumentar a absorção de P lábil no solo (P-resina e Pi-NaHCO 3 ). Quanto às frações pouco lábeis, observa-se que plantas micorrizadas absorveram mais a fração Pi-NaOH. Em muitos estudos, plantas micorrizadas e não micorrizadas parecem usar a mesma fonte de P lábil, entretanto Jayachandran et al. (1989) mostraram que plantas micorrizadas podem obter $\mathrm{P}$ de fontes menos disponíveis. Na braquiária e soja, a absorção de Po-NaOH nas plantas micorrizadas foi maior, sugerindo que a mineralização de Po é aumentada por fungos micorrízicos, e a produção de fosfatase poderia ser um mecanismo direto para a mineralização de Po no solo (Jayachandran et al., 1992). Fica evidente que a influência das micorrizas na distribuição das diferentes frações de $\mathrm{P}$ no solo é muito comple$\mathrm{xa}$, pois depende de fatores relacionados ao uso e manejo do solo.

\section{Conclusões}

1. No Latossolo Amarelo coeso e Latossolo Vermelho ácrico, os teores de P-Al, P-Fe e P-Ca são mai- ores nos solos cultivados; no Latossolo Vermelho distroférrico, as frações P-Al e P-Fe são maiores no solo não cultivado.

2. Com a adição de P, as frações Pi-resina, $\mathrm{Pi}-\mathrm{NaHCO}_{3}$ e $\mathrm{Pi}-\mathrm{NaOH}$ são aumentadas, sendo as maiores proporções do $\mathrm{P}$ adicionado encontradas na fração Pi-NaOH.

3. As frações de P nos solos são pouco influenciadas pela micorriza.

4. Os solos cultivados apresentam menores teores de $\mathrm{P}$ nas frações orgânicas do que os não cultivados.

5. Nos solos cultivados com braquiária, verificamse menores teores de $\mathrm{P}$ nas frações pouco lábeis e maiores nas frações mais lábeis.

\section{Referências}

ALVES, G. L. N. Micorriza vesicular-arbusculares no crescimento e utilização do fósforo do solo pela braquiária e estilosantes. 1988. 42 f. Dissertação (Mestrado em Solos e Nutrição de Plantas) - Universidade Federal de Lavras, Lavras, 1988.

ARAÚJO, M. S. B.; SALCEDO, I. H. Formas preferenciais de acumulação de fósforo em solos cultivados com cana-de-açúcar na Região Nordeste. Revista Brasileira de Ciência do Solo, Campinas, v. 21, n. 4, p. 643-650, out./dez. 1997

ARAÚJO, M. S. B.; SALCEDO, I. H.; SAMPAIO, E. V. S. B. Efeito de fertilizações fosfatadas anuais em solo cultivado com cana-de-açúcar, intensidade e formas de acumulação. Revista Brasileira de Ciência do Solo, Campinas, v. 17, n. 3, p. 389-396, set./out. 1993.

BAHIA FILHO, A. F. C.; BRAGA, J. M. Fósforo em latossolo do Estado de Minas Gerais - II: quantidade de fósforo disponível. Revista Ceres, Viçosa, MG, v. 22, n. 119, p. 50-61, jan./fev. 1975.

BECK, M. A. Inorganic and organic phosphorus transformations during 18 years of cultivation in the Amazon basin. 1991. 145 leaves. Dissertation (Master in Soil Science) - North Caroline State University, Raleigh, 1991.

BECK, M. A.; SANCHEZ, P. A. Soil phosphorus fraction dynamics during 18 years of cultivation on a Typic Paleudult. Soil Science Society of America Journal, Madison, v. 58, n. 5, p. 1424-1431, Sept./Oct. 1994. 
CASSAGNE, N.; REMAURY, M. Forms and profile distribution of soil phosphorus in alpine Inceptisols and Spodosols (Pyrenees, France). Geoderma, Amsterdam, v. 95, n. 1/2, p. 161-172, Mar. 2000.

CHANG, S. C.; JACKSON, M. L. Fractionation of soil phosphorus. Soil Science, Baltimore, v. 84, n. 1, p. 133-144, July 1957.

CROSS, A. F.; SCHLESINGER, W. H. A literature review and evaluation of the Hedley fractionation: applications to the biogeochemical cycle of soil phosphorus in natural ecosystems. Geoderma, Amsterdam, v. 64, n. 3/4, p. 197-214, Jan. 1995.

DAROUB, S. H.; PIERCE, F. J.; ELLIS, B. G. Phosphorus fractions and fate of phosphorus-33 in soils under plowing and no-tillage. Soil Science Society of America Journal, Madison, v. 64, n. 1, p. 170-176, Jan./Feb. 2000.

DAY, P. R. Particle fractionation and particle-size analysis. In: BLACK, C. A. (Ed.). Methods of soil analysis. Madison: American Society of Agronomy, 1965. v. 1, p. 545-556.

DUFFERA, M.; ROBARGE, W. P. Characterization of organic and inorganic phosphorus in the highland plateau soils of Ethiopia. Communications in Soil Science and Plant Analysis, New York, v. 27, n. 15/17, p. 2799-2814, 1996.

EMBRAPA. Centro Nacional de Pesquisa de Solos (Rio de Janeiro, RJ). Manual de métodos de análise do solo. Rio de Janeiro, 1997. 212 p.

GERDEMANN, J. W.; NICOLSON, T. H. Spores of mycorrhizal endogone species extracted from soil by wet sieving and decanting. Transactions of the British Mycological Society, London, v. 46, n. 2, p. 235-244, 1963.

HEDLEY, M. J.; STEWARD, W. B.; CHAUHAN, B. S. Changes in inorganic and organic soil phosphorus fraction induced by cultivation practices and laboratory incubation. Soil Science Society of America Journal, Madison, v. 46, n. 5, p. 970-976, Sept./Oct. 1982.

HSU, P. H. Aluminium oxides and oxyhydroxids. In: DIXON, J. B.; WEED, S. B. (Ed.). Minerals in soil environments. Madison: Soil Science Society of America, 1977. p. 99-144.

JAYACHANDRAN, K.; SCHWAB, A. P.; HETRICK, B. A. D. Mineralization of organic phosphorus by vesicular-arbuscular mycorrhizal fungi. Soil Biology and Biochemistry, Oxford, v. 24, n. 9, p. 897-903, Sept. 1992.
JAYACHANDRAN, K.; SCHWAB, A. P.; HETRICK, B. A. D. Mycorrhizal mediation of phosphorus availability: synthetic iron chelate effects on phosphorus solubilization. Soil Science Society of America Journal, v. 53, p. 1701-1706, 1989.

LILIENFEIN, J.; WILCKE, W.; AYARZA, M. A.; VILELA, L. Chemical fractionation of phosphorus, sulphur, and molybdenum in Brazilian savannah Oxisols under different land use. Geoderma, Amsterdam, v. 96 , n. 1/2, p. 31-46, May 2000

MURPHY, J.; RILEY, J. P. A modified single solution methods for the determination of phosphate in natural waters. Analytica Chimica Acta, Amsterdam, v. 26, n. 1, p. 31-36, 1962.

NOVELINO, J. O. Disponibilidade de fósforo e sua cinética, em solos sob cerrado fertilizados com fósforo, avaliada por diferentes métodos de extração. 1999. 70 f. Tese (Doutorado em Solos e Nutrição de Plantas) Universidade Federal de Viçosa, Viçosa, MG, 1999.

RAIJ, B. van; FEITOSA, C. T. Correlação entre o P extraído de solo por diversos extratores químicos e o absorvido pelo milho. Bragantia, Campinas, v. 39, n. 1, p. 51-57, 1980.

RHEINHEIMER, D. S.; ANGHINONI, I.; KAMINSKI, J. Depleção do fósforo inorgânico de diferentes frações provocada pela extração sucessiva com resina em diferentes solos e manejos. Revista Brasileira de Ciência do Solo, Viçosa, MG, v. 24, n. 2, p. 345-354, abr./jun. 2000.

SAGGIN-JÚNIOR, O. J.; SIQUEIRA, J. O.; GUIMARÃES, P. T. G.; OLIVEIRA, E. Interação fungos micorrízicos versus superfosfato e seus efeitos no crescimento e teores de nutrientes do cafeeiro em solo não fumigado. Revista Brasileira de Ciência do Solo, Campinas, v. 18, n. 1, p. 27-36, jan./jun. 1994.

SCHMIDT, J. P.; BUOL, S. W.; KAMPRATH, E. J. Soil phosphorus dynamics during 17 years of continuous cultivation: a method to estimate long-term $\mathrm{P}$ availability. Geoderma, Amsterdam, v. 78, n. 1/2, p. 59-70, July 1997.

SIQUEIRA, J. O. Micorrizas arbusculares. In: ARAUJO, R. S.; HUNGRIA, M. (Ed.). Microrganismos de importância agrícola. Brasília: Embrapa-CNPAF/ Embrapa-CNPSo, 1994. p. 151-194.

STEWART, J. W. B.; HEDLEY, J. M.; CHAUHAN, B. S. The immobilization, mineralization and redistribution of 
phosphorus in soils. In: WESTERN CANADA PHOSPHATE SYMPOSIUM, 1980, Alberta. Proceedings... Alberta: Soil Science Society of America, 1980. p. 276-306.

TIESSEN, H.; SALCEDO, I. H.; SAMPAIO, V. S. B. Nutrient and soil organic matter dynamics under shifting cultivation in semi-arid Northeastern Brazil. Agriculture Ecosystems Environment, Amsterdam, v. 38, n. 3, p. 139-151, Feb. 1992.

TIESSEN, H.; STEWART, J. W. B.; COLE, C. V. Pathways of phosphorus transformations in soils of differing pedogenesis. Soil Science Society of America Journal, Madison, v. 48, n. 4, p. 853-858, July/Aug. 1984.

WILLIAMS, J. D. H.; MAYERS, T.; NRIAGU, J. O. Extractability of phosphorus from phosphate minerals common in soils and sediments. Soil Science Society of America Journal, Madison, v. 44, n. 3, p. 462-465, May/ June 1980.

WRIGHT, C. J.; COLEMAN, D. C. The effects of disturbance events on labile phosphorus fractions and total organic phosphorus in the southern Appalachians. Soil Science, Baltimore, v. 164, n. 6, p. 391-402, June 1999. 